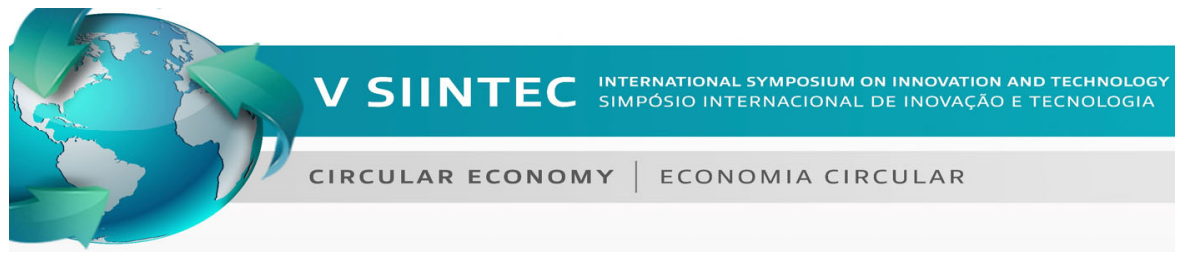

\title{
FERRAMENTAS DE SUPORTE A DECISÃO PARA REDES DE TRATAMENTO DE ÁGUAS RESIDUAIS.
}

Leonardo Oliveira Santos de Santana ${ }^{1}$, Edna dos Santos Almeida1 ${ }^{1}$ Ewerton Emmanuel da Silva Calixto ${ }^{1}$, Fernando Luiz Pellegrini Pessoa ${ }^{1}$, Cristina Carvalho ${ }^{1}$

Centro universitário SENAI-CIMATEC; Salvador/Ba; leosantana049@gmail.com

Resumo: Ferramentas de suporte à decisão (DSSs) são cada vez mais comuns no desenvolvimento de redes de tratamento e reuso de água pois auxiliam em problemas de síntese e simulação dos processos de tratamento. O presente trabalho apresenta um levantamento dos principais aspectos e passos a serem realizados no desenvolvimento de uma DSS, por meio de uma revisão bibliográfica em bases de periódicos. A fim de contornar o problema relacionado à explosão combinatória na etapa da síntese. Os artigos levantados sugerem projetos de DSSs que utilizam métodos intuitivos, como o método heurístico, aliadas ao uso de programação matemática em seu desenvolvimento, assim como regras para otimização no que diz respeito ao sequenciamento e mistura das correntes de efluentes.

Palavras-Chave: Ferramentas de suporte à decisão, rede de reuso de água, síntese de processos, regras heurísticas.

\section{DECISION SUPPORT SYSTEMS FOR WASTEWATER TREATMENT PROCESSES.}

\begin{abstract}
Decision support tools (DSSs) are very commons in the development of Wastewater treatment trains and reuse networks, this DSSs assist in the simulation of this treatment process. This paper presents a survey of the main aspects and steps to be performed in the development of an DSS, through a bibliographic review in journal bases. To solve the blast-related problem combined in the Synthesis step. The articles raised suggest designs of DSSs that use intuitive methods, such as the heuristic method, combined with the use of mathematical programming, as well as optimization rules regarding the sequencing and mixing of effluent variables.
\end{abstract}

Keywords: Decision support systems, water reuse network, Process synthesis, Heuristics rules. 


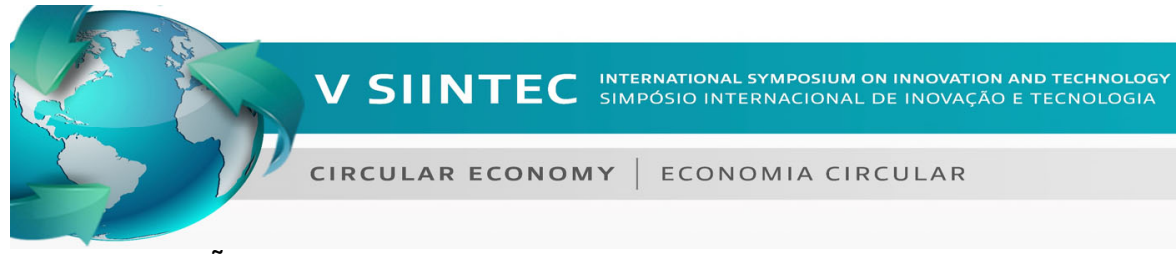

\section{INTRODUÇÃO}

A atividade industrial consome uma grande quantidade de recursos hídricos e produz uma grande quantidade de contaminantes na forma de efluentes aquosos. Por muitos anos perpetuou-se a visão de que tais efluentes, após tratamento, devem ser dispostos diretamente num manancial hídrico (visão fim de tubo).

A água sempre foi vista como um recurso abundante, sem haver a devida preocupação no tratamento dos efluentes descartados, causando, como consequência, impactos ao meio ambiente. Atualmente os conceitos de economia de base tripla, ou de sustentabilidade, estão mudando este paradigma, de forma que recursos como a água são utilizados de maneira mais consciente e os efluentes são devidamente tratados a fim de não causarem grandes impactos ao meio ambiente. [1]

Nesse contexto existem pelo menos seis desafios e oportunidades para o tratamento de efluentes domésticos e industriais, todos pautados nos conceitos de linha de base tripla (sustentabilidade): (1) Gerenciamento de recursos hídricos; (2) Necessidade de projetar novas estações de tratamento de efluentes para a recuperação de energia e outros recursos; (3) Necessidade de produzir um efluente tratado adequado para a produção de água potável; (4) A implantação de sistemas descentralizados de tratamento; (5) Gerenciamento integrado dos efluentes;(6) Utilização de técnicas de desenvolvimento de baixo impacto..$^{[1,2]}$

Assim, as redes de reuso de água e efluentes representam uma oportunidade na questão do desenvolvimento sustentável, pois não só incluem a coleta/ distribuição, mas também o tratamento do efluente industrial. Krovvidy et al. ${ }^{[3]}$ propõem uma abordagem em duas fases para o projeto de sistemas de tratamento de efluentes. $\mathrm{Na}$ primeira fase, etapa de análise, há o desenvolvimento de um sistema de aprendizagem para gerar regras de conhecimento a partir de uma base de dados da tratabilidade do efluente. Na segunda fase, chamada fase de síntese, onde se desenvolveu duas metodologias diferentes: uma abordagem de busca heurística e uma abordagem de rede neural artificial para gerar sistema de tratamento com custo mínimo. A função de pesquisa heurística é desenvolvida com base nas capacidades de remoção dos processos de tratamento. ${ }^{[3]}$

\section{1- Síntese de processos e os DSSs}

A síntese é a etapa nobre de um projeto de processos, na qual se definem os equipamentos e a forma como eles se conectam para formar um determinado fluxograma. Nos diversos processos industriais, o arranjo dos equipamentos pode variar bastante e crescer em escala proibitiva com o número de equipamentos necessários (explosão combinatória), gerando diversos fluxogramas plausíveis, onde cada um desses fluxogramas representa uma solução viável do problema de síntese. ${ }^{[4]}$

A dificuldade causada pela explosão combinatória (Ver Tabela 1) na síntese pode ser resolvida por meio de métodos intuitivos, ou baseados em conhecimento (KBS), tais como os métodos heurísticos. Esses sistemas KBS tentam simular um cérebro humano resolvendo problemas, usando fontes de conhecimento, dentro de um domínio concreto. Além disso, tais sistemas são baseados em conhecimentos 


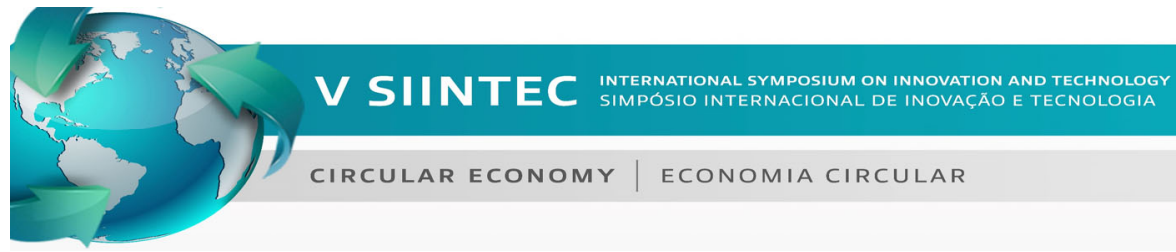

formados por um conjunto de informações e relações declarativas ou processuais, bem como possuem certas heurísticas que formam o corpo de conhecimento. ${ }^{[5]}$

Tabela 1: Crescimento do número de fluxogramas em função do número de processos e contaminantes (Explosão combinatória).

\begin{tabular}{|c|c|c|c|}
\hline Processos & 1 & 2 & 3 \\
\hline Contaminantes & & 2 & 3 \\
\hline 2 & 1 & 8 & 18 \\
\hline 3 & 2 & 40 & 135 \\
\hline 4 & 5 & 224 & 1134 \\
\hline 5 & 14 & 1344 & 10206 \\
\hline 6 & 42 & 8448 & 96228 \\
\hline 7 & 132 & 54912 & 938223 \\
\hline 8 & 429 & & \\
\hline
\end{tabular}

Fonte: PERLINGEIRO, 2005.

Geralmente uma grande quantidade de conhecimento é necessária para resolver este tipo de problema. O corpo de conhecimento é codificado em forma de regras de inferência que possuem a seguinte estrutura:

\section{SE <condição> ENTÃO <ação>}

Dentre os métodos baseados em conhecimento existe o método heurístico, que consiste em utilizar regras práticas baseadas na experiência acumulada na resolução de um determinado tipo de problema. O método foi identificado e formalizado pela inteligência artificial. Este é um método de decisões sequenciais em que o sistema é montado progressivamente como fruto de uma sequência de decisões. O método heurístico não conduz à uma solução ótima, mas dependendo da qualidade das regras pode-se fornecer uma solução próxima da ótima. Este método possui como principal vantagem a rapidez na busca da solução, uma vez que ignora as demais soluções viáveis, contornando o problema da explosão combinatória. ${ }^{[4]}$

O auxilio computacional na síntese de processos teve grande crescimento recentemente, principalmente com o surgimento de ferramentas computacionais com interface amigável ao usuário. Tais softwares ou ferramentas de suporte à decisão, fazem uso de metodologias diversas, tais como: programação matemática e métodos intuitivos. Uma ferramenta de suporte à decisão é um sistema de informação que ajuda um usuário na escolha da melhor solução para um dado problema. As DSSs são muito aplicadas em problemas envolvendo o tratamento de efluentes aquosos, incluindo a seleção e projetos de processos de tratamento, sequenciamento (em série ou em paralelo) do tratamento e no monitoramento e controle dessas plantas. A maior parte das ferramentas de decisão existentes emprega conhecimento heurístico e consideram apenas os principais fatores técnicos e econômicos da seleção de um processo de tratamento de efluentes, como a eficiência de remoção de contaminantes e custo de capital. As Figuras 1 e 2 abaixo mostram um esquema genérico de uma rede de reuso e um exemplo de síntese de processo de tratamento de efluente respectivamente. ${ }^{[6]}$ 


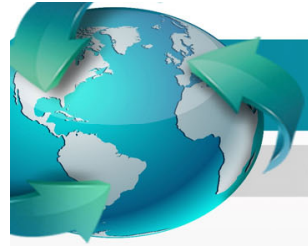

V SIINTEC

CIRCULAR ECONOMY | ECONOMIA CIRCULAR

Figura 1. Rede de reuso genérica.

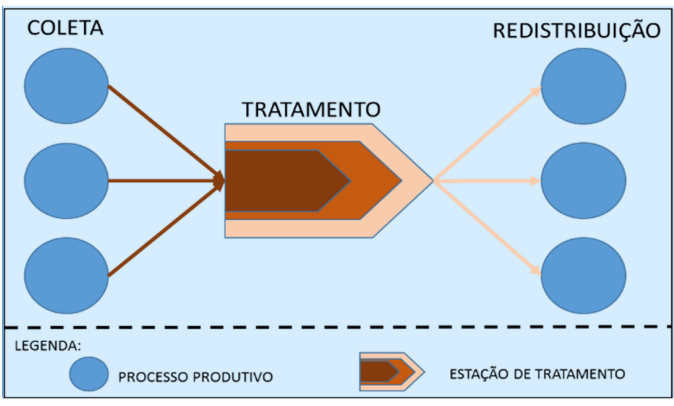

Fonte: Propria, 2019

Figura 02: Síntese de processo ótimo de tratamento de efluente gerado por uma DSS.

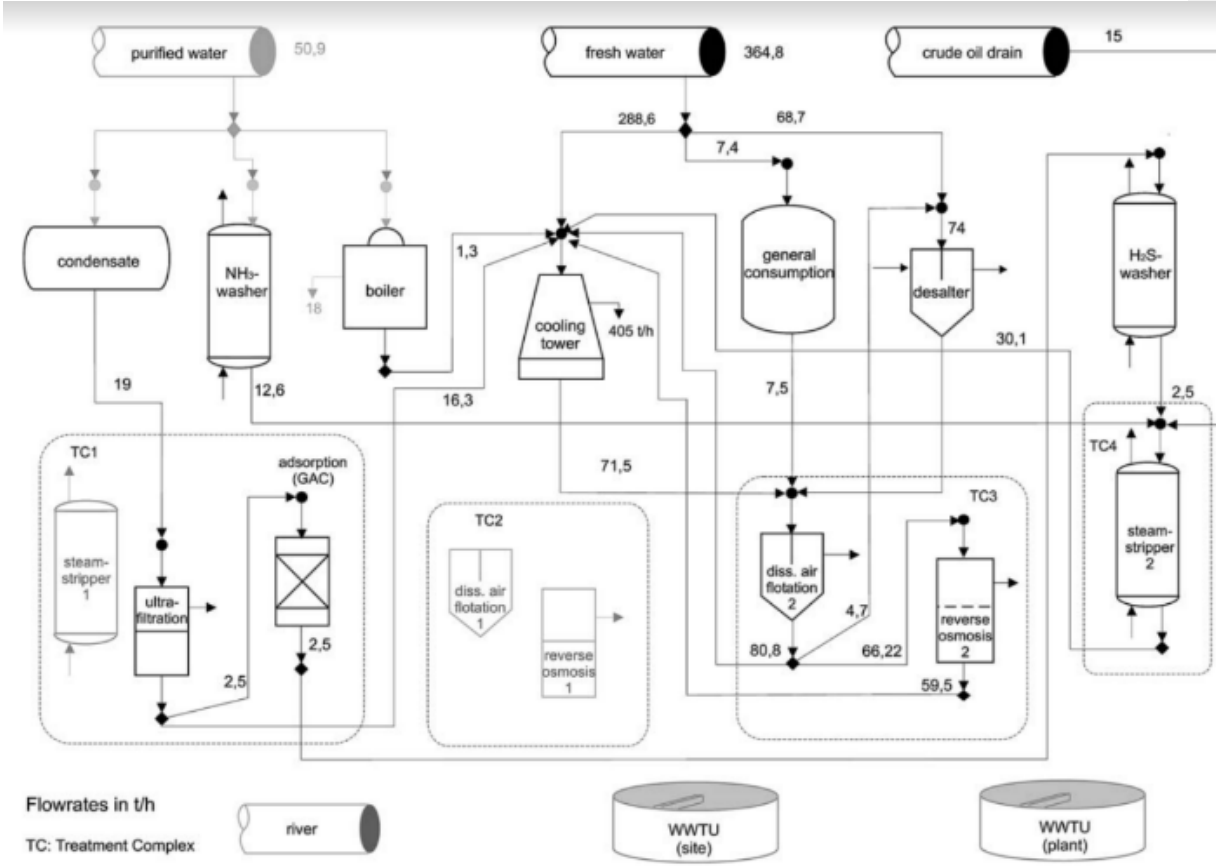

Fonte: ULLMER, 2005

Este artigo tem por objetivo levantar informações na literatura acerca do sequenciamento recomendado na organização de um projeto de uma ferramenta DSS a partir de um levantamento bibliográfico dos trabalhos recentemente publicadas sobre o tema.
Comentado [EEdSC3]: Inserir legenda automática pelo word. Colocar acima da figura. Nova regra ABNT.

Tem que explicar a figura. Descrever o que quer dizer com a figura.

Comentado [EdSA4]: Seria distribuição e não REdistribuição
Comentado [EEdSC5]: Inserir legenda automática pelo word Colocar acima da figura. Nova regra ABNT.

Tem que explicar a figura. Descrever o que quer dizer com a figura. 


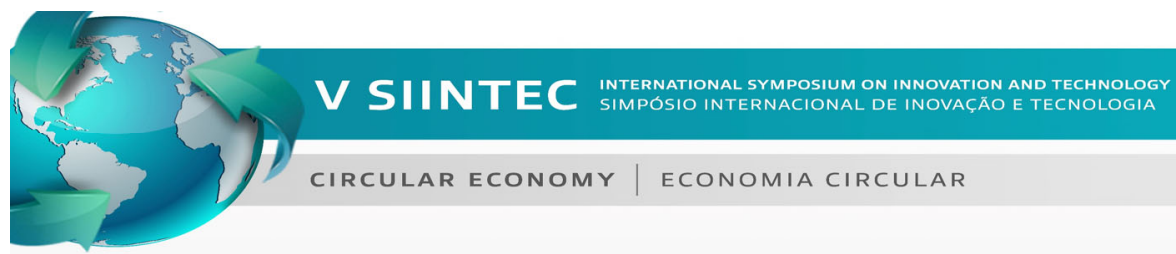

\section{METODOLOGIA}

A pesquisa foi realizada nas bases de pesquisa dos periódicos Web of Science e Google Acadêmico. Utilizou-se na pesquisa a combinação das seguintes palavraschave: "Heuristic Rule or Heuristic Search", "Knowledge based system and wastewater treatment", "Rule based systems and Decision support systems", entre outras combinações. O período de busca foi delimitado nos últimos dez anos

Nos resultados obtidos da busca de artigo nas bases de dados foram aplicados os seguintes critérios de exclusão: publicações que não tinham o artigo completo disponível, aderência aos objetivos da pesquisa e duplicidade nas diferentes plataformas. A partir dessas buscas, e após a aplicação dos critérios de exclusão, prosseguiu-se com das metodologias de desenvolvimento das redes de tratamento de efluentes. Foram analisados um total de 6 artigos, que continham o passo a passo da elaboração de uma ferramenta DSS.

\section{RESULTADOS E DISCUSSÃO}

A revisão bibliográfica do tema levantou cerca de 6 publicações que descrevem metodologias de desenvolvimento de ferramentas de suporte à decisão, principalmente aquelas relacionadas aos sistemas de tratamento de efluentes industriais. Uma rede de reuso de efluentes inclui os sistemas de coleta do efluente bruto, tratamento e distribuição do efluente tratado próprio para consumo industrial. Uma DSS deve levar em conta além de heurísticas para organização do processo, fatores e regras para síntese da rede de tratamento.

\section{1- Etapas de desenvolvimento de uma DSS}

Os estágios de desenvolvimento de uma DSS são parecidos na maioria dos casos independentemente da aplicação. A Figura 3 representa os quatro estágios para o desenvolvimento de uma DSS para um problema de tratamento de água [6]. Segundo o autor, o primeiro estágio consiste na análise e interpretação do problema em questão (pode-se fazer uma análise genérica, onde diferentes processos são considerados para remover vários contaminantes). No segundo estágio, o conhecimento obtido no primeiro pode ser representado numericamente, ou em forma heurística de regra geral. No terceiro estágio há o suporte real à decisão, em que as alternativas são propostas e avaliadas, e a seleção e o projeto do processo ocorrem. Nesta etapa os métodos de otimização desempenham um papel importante na incorporação de todos os fatores para chegar à solução ótima do problema. A quarta etapa garante a usabilidade, validando e verificando a lógica do DSS, assim como melhorando a interatividade do usuário com o DSS desenvolvido.

De forma semelhante Ullmer et al ${ }^{[7]}$ propõe também uma estratégia de solução para o tratamento de efluentes. A solução foi dividida em várias etapas, nas 4 primeiras, regras heurísticas são utilizadas na aquisição de descrições mais apropriadas para o design da rede de tratamento. No final, a solução ótima foi obtida por meio de computação e analisada usando modelos matemáticos e outros métodos. ${ }^{[6,7]}$ 


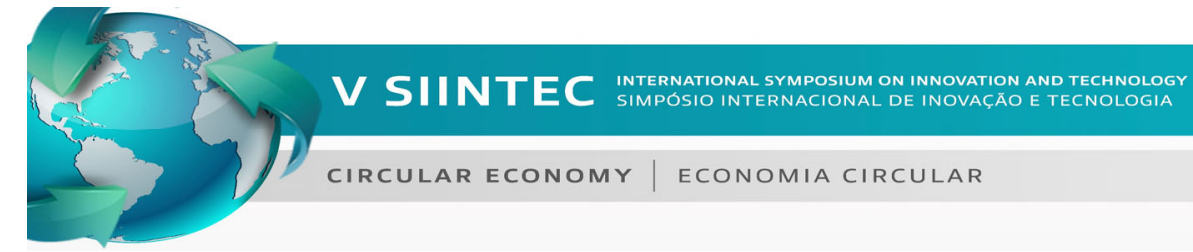

Figura 3: Etapas do desenvolvimento de um sistema de apoio à decisão de tratamento de água.

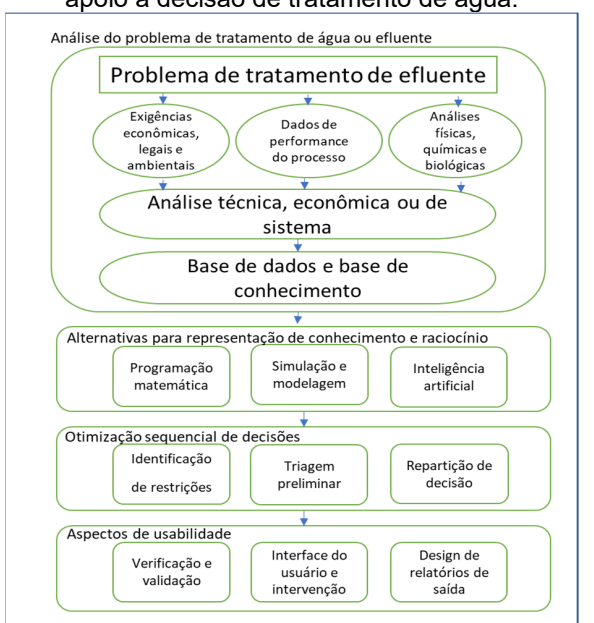

Fonte: HAMOUDA, 2009
Figura 4: Metodologia de solução

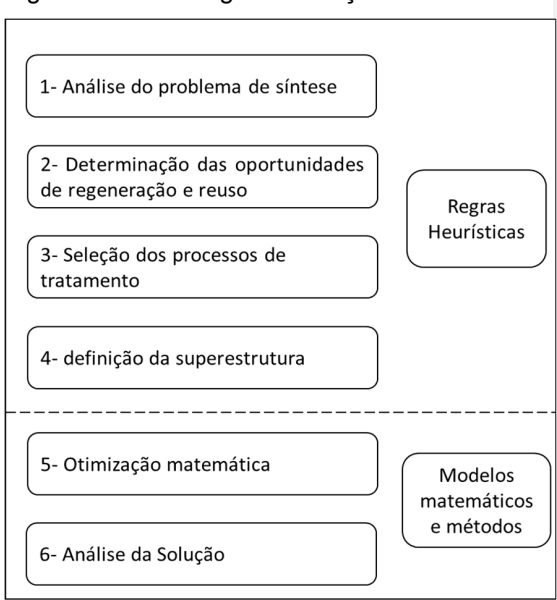

Fonte: ULLMER, 2005

Nas primeiras etapas se faz necessário encontrar uma descrição apropriada do problema de síntese além da determinação dos locais onde reuso e regeneração são possíveis e a sequência de operações unitárias do processo. Para isto se faz necessário o conhecimento das características do efluente na entrada de cada operação e a taxa de remoção que se deseja alcançar. Na quarta etapa é feita a inclusão de algumas regras que limitem e proíbam certas combinações de equipamentos levando a uma detecção mais sensata da solução. Esta etapa é ainda baseada em regras heurísticas, assim sendo, as regras e restrições devem ser inseridas com muito cuidado para que não seja excluída uma boa solução para o problema. Por fim, uma otimização matemática pode ser realizada minimizando os custos totais fixos, variados, de investimento e operação. O sexto passo consiste na análise da solução apresentada pelo DSS. ${ }^{[7]}$

\section{2- Procedimentos de projeto propostos na literatura}

Dentro do processo de desenvolvimento de uma DSS, existe o a etapa de projeto de uma rede de tratamento de efluentes (WWTN), que está incluída como uma das etapas no fluxo das redes de reuso, alguns autores contribuíram com algoritmos simples para essa finalidade, levando em conta os aspectos listados por Alnouri, 2014, Alnouri et al Alnouri et al 2016. ${ }^{[8,9,10]}$

A minimização da mistura desnecessária de correntes com diferentes concentrações de contaminantes recebe uma atenção especial de $\mathrm{LI}$, et al ${ }^{[11]}$. O autor propõe um algoritmo para a elaboração de um processo de tratamento de efluentes distribuído com múltiplos contaminantes. Nesses sistemas é importante minimizar a mistura desnecessária das correntes a fim de reduzir ao máximo a taxa total de fluxo de tratamento, uma vez que a mistura desnecessária reduz as concentrações de contaminantes, podendo aumentar o custo de tratamento. Com base nisso, regras heurísticas são propostas para evitar essa mistura desnecessária [11].

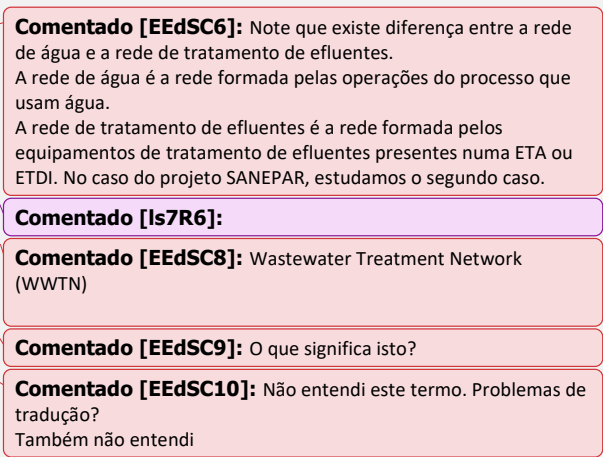




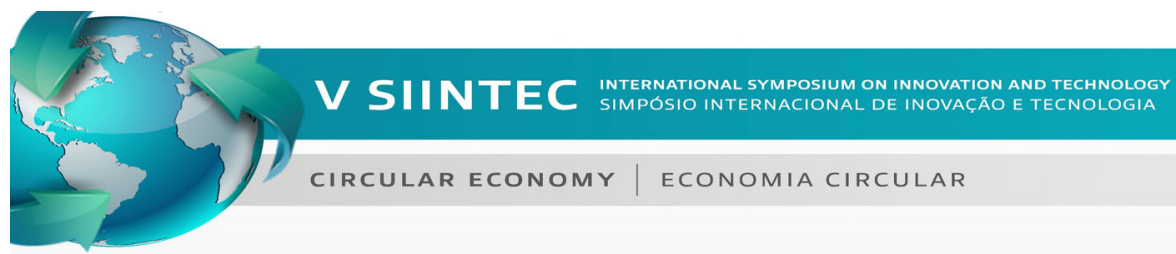

Para a elaboração da sequência de operações do tratamento são dadas as seguintes informações: (i) um conjunto de fluxos de águas residuais contendo múltiplos contaminantes com vazões conhecidas e concentrações de contaminantes; (ii) um conjunto de processos de tratamento disponíveis, sendo cada um capaz de remover um ou alguns contaminantes; (iii) as restrições máximas de concentração de entrada e proporções de remoção de cada processo de tratamento; (iv) a regulação ambiental de cada contaminante. O objetivo é minimizar a vazão de efluente encaminhado para descarte e o número de processos de tratamento de uma WWTN distribuída sob a premissa de atender à regulação ambiental de cada contaminante. Assume-se que cada processo só pode ser usado uma vez e não há perda de água durante as operações de tratamento. ${ }^{[11]}$

Outro procedimento de projeto é feito em dois estágios (Figura 6). Na primeira etapa tem-se a construção inicial da rede, que inclui o cálculo da carga de massa mínima de remoção por contaminante e identificação das contaminantes chaves para cada processo; cálculo do TMIP (valor do potencial total de influência da mistura). Se a carga removida for maior ou igual que o valor de massa mínima da contaminante chave pode-se seguir para o segundo estágio; se não, retorna-se para "b". A segunda etapa é composta pelo ajuste da rede inicial para a obtenção do design final, quando a carga de contaminante precisa ser removida por mais de um processo. ${ }^{[11,12]}$
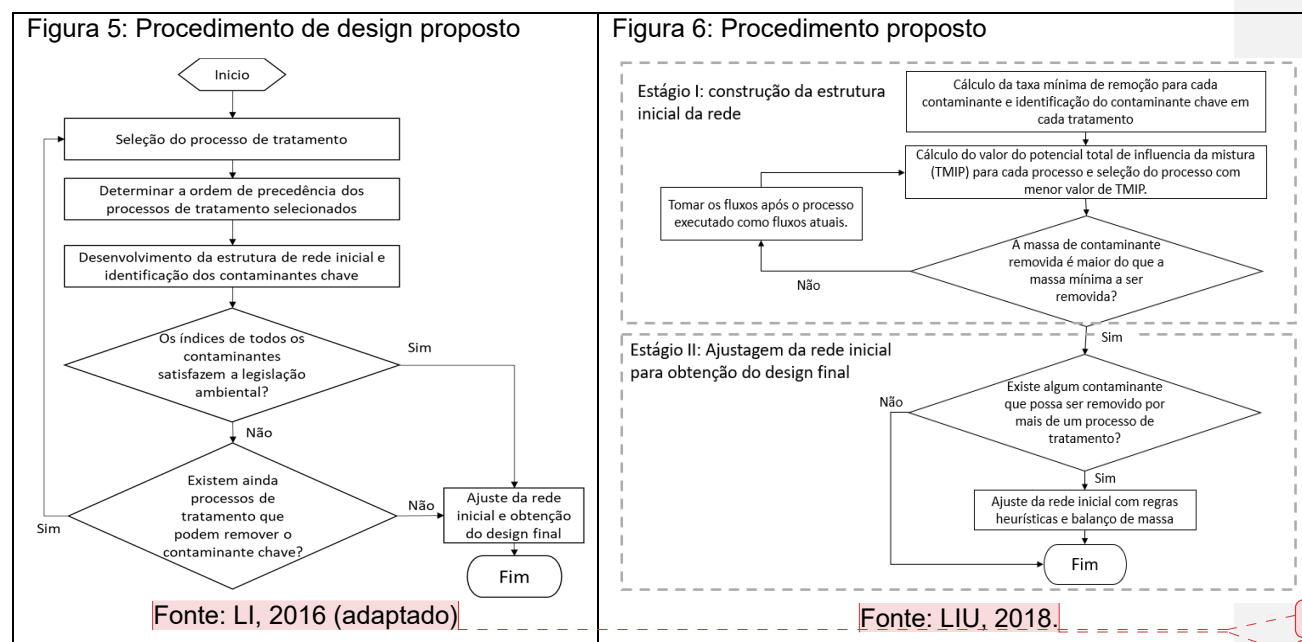

\section{CONCLUSÃO}

A partir dos artigos levantados pôde-se inferir que redes de reuso de efluentes representam um dos desafios e oportunidades no cenário do desenvolvimento sustentável. Com o auxílio delas pode-se reduzir consumo de água como insumo e minimizar impactos ambientais causados pelo lançamento de efluentes aquosos em corpos hídricos. Todavia um dos desafios intrínsecos à elaboração de uma rede de reuso de água completa é o desenvolvimento de uma rede ótima, que otimiza a coleta e a redistribuição da água residual, assim como o processo de tratamento dentro das ETEs. 


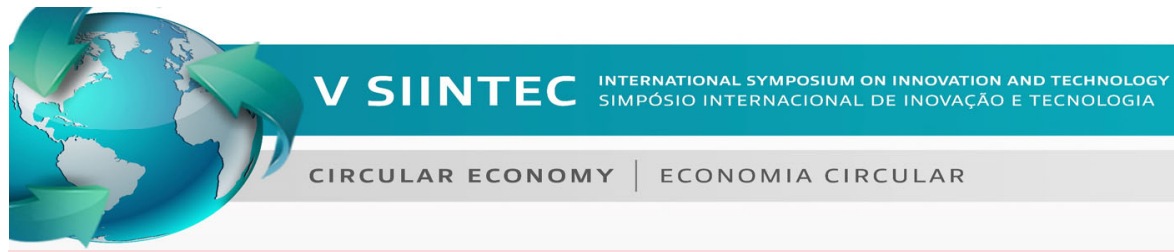

Com o auxílio da informática e da programação temos as chamadas ferramentas de suporte a decisão (DSS), softwares que são feitos para auxiliar o usuário na síntese e simulação desses processos. O processo de desenvolvimento de uma ferramenta DSS envolve a parte de uma pesquisa inicial e geral do tema, determinação das oportunidades de reuso, aquisição de dados de qualidade do efluente, e seleção preliminar dos processos de tratamento. Para resolver o problema da explosão combinatória se faz necessário o uso de sistemas baseados em conhecimento e inteligência artificial, com o auxílio de métodos intuitivos, como o método heurístico. A partir do uso dessas regras, e definida a superestrutura, parte-se para a modelagem matemática (programação linear e não linear por exemplo). Por fim será feita a análise do fluxograma gerado. Dessa forma os resultados obtidos na pesquisa mostraram um caminho seguro na direção do desenvolvimento de uma ferramenta DSS.

\section{REFERÊNCIAS}

${ }^{1}$ MIERZWA, J. C; HESPANHOL, I. Água na indústria: uso racional e reuso [1 ${ }^{\text {a }}$ reimpr.] - São Paulo, oficina dos textos,2017.

2 METCALF \& EDDY. Tratamento de efluentes e recuperação de recursos; tradução: Ivanildo Hespanhol, José Carlos Mierzwa. - 5. Ed.- Porto Alegre: AMGH,2016.

${ }^{3}$ KROVVIDY, S., WEE, W., SUMMERS, R., \& COLEMAN, J. (1991). An al approach for wastewater treatment systems. Journal of applied intelligence, pp. 247-261.

${ }^{4}$ PERLINGEIRO, C.A.G.; Engenharia de processos: análise, simulação, otimização e síntese de processos químicos. 2. Ed.- Rio de Janeiro: Editora Blucher, 2005.

${ }^{5}$ MARRĖ, M. S., CORTÉS, u., \& LAFUENTE, J. (s.d.). Knowledge-based techniques in wastewater treatment plants management.

${ }^{6}$ HAMOUDA, M. A., ANDERSON, W., \& HUCK, P. (2009). Decision support systems in water and wastewater treatment process selection and design: a review. Water Science \& Technology-WST.

7 ULLMER, C., KUNDE, N., LASSAHN, A., GRUHN, G., \& SCHULZ, K. (2005). WADO: water design optimization- methodology and software for the synthesis of process water systems. Journal of cleaner production, pp. 485-494.

${ }^{8}$ Alnouri, S. Y.; Linke, P.; El-Halwagi, M. M. Synthesis of industrial park water reuse networks considering treatment systems and merged connectivity options. 2016

${ }^{9}$ Alnouri, S. Y.; Linke, P.; El-Halwagi, M. M. A synthesis approach for industrial city water reuse networks considering central and distributed treatment systems. 2014

${ }^{10}$ ALNOURI, S.Y; LINKE, P; EL-HALWAGI, M. Water integration in industrial zones: a spatial representation with direct recycle applications. 2015

11 LI, A., ZHANG, J., \& LIU, Z. (2016). Design of distributed wastewater treatment networks of multiple contaminants with maximum inlet concentration. Journal of cleaner production, pp. 170-178.

12 LIU, C., LI, A., KLEMES, J., \& LIU, Z. (2018). Design of distributed wastewater treatment networks by combining total mixing influence potential indicator with heuristic rules. Journal of cleaner production, pp. 604-613.

Comentado [EEdSC13]: Não está claro qual foi a conclusão do
seu trabalho.
Por exemplo:
"Com base na revisão realizada pode-se concluir que...."
Leo, precisamos conversar melhor sobre este artigo....
Concordo com Ewerton

